\title{
Algumas desigualdades úteis e o teste da segunda derivada
}

\author{
Eddy Kwessi (D)
}

Seth Kermausuor

Geraldo de Souza

\section{Resumo}

Determinar os pontos de máximo e mínimo locais de uma função de duas variáveis $\mathrm{z}=\mathrm{f}(\mathrm{x}, \mathrm{y})$ em um curso de cálculo requer o uso do conhecido "o teste das segundas derivadas", usualmente ensinado aos estudantes nos primeiros anos do curso superior. Em particular, os cursos de engenharia, física e matemática. Em nossa experiência ensinando cálculo, usualmente escolhemos exemplos bem simples para ilustrar o uso desse teste. Em relação aos exemplos e aplicações mais complicados, procuramos evitá-los para facilitar o aprendizado e compreensão dos estudantes.

O nosso objetivo nestas notas é apresentar, aos leitores interessados, casos patológicos do teste das segundas derivadas, e ilustrar a importância dele em conexão com várias desigualdades. Tais desigualdades, são frequentemente vistas nos primeiros cursos de análise e álgebra linear. Por exemplo, as desigualdades de Cauchy-Schwarz-Bunyakowski, de Young, de Bernoulli e a desigualdade da média aritmética e geométrica.

O teste das segundas derivadas para funções de duas variáveis $\mathrm{z}=\mathrm{f}(\mathrm{x}, \mathrm{y})$ envolve uma expressão usualmente denotada por $\mathrm{D}$ que é definida por $\mathrm{D}=\mathrm{D}(\mathrm{a}, \mathrm{b})=\mathrm{f}_{\mathrm{xx}}(\mathrm{a}, \mathrm{b}) \cdot \mathrm{f}_{\mathrm{yy}}(\mathrm{a}, \mathrm{b})-\left(\mathrm{f}_{\mathrm{xy}}(\mathrm{a}, \mathrm{b})\right)^{2}$, sendo que o ponto $\mathrm{P}=(\mathrm{a}, \mathrm{b})$ é candidato a máximo local, mínimo local ou ponto de sela, e as funções $\mathrm{f}_{\mathrm{xx}}, \mathrm{f}_{\mathrm{yy}}$ e $\mathrm{f}_{\mathrm{xy}}$ são as segundas derivadas parciais de $\mathrm{f}(\mathrm{x}, \mathrm{y})$. O teste usa as seguintes condições em $\mathrm{D}(\mathrm{a}, \mathrm{b})$ :

1. $\mathrm{D}>0$

2. $\mathrm{D}<0$

3. $\mathrm{D}=0$.

No caso 1 , o ponto $\mathrm{P}=(\mathrm{a}, \mathrm{b})$ pode ser de máximo ou mínimo, no caso 2 ele é um ponto de sela e, finalmente, no caso 3 o teste é inconclusivo.

Nestas notas vamos mostrar que o caso 3 , em geral, não é inconclusivo. Por exemplo, considere a desigualdade de Cauchy-Schwarz-Bunyakowski que diz, se $\mathrm{X}=(\mathrm{x}, \mathrm{y})$ e $\mathrm{Y}=(\alpha, \beta)$, então o produto interno $\langle\mathrm{X}, \mathrm{Y}\rangle$ e as normas em $\mathbb{R}^{2}$ de $\mathrm{X}$ e $\mathrm{Y}$ são relacionadas pela desigualdade

$$
|\langle\mathrm{X}, \mathrm{Y}\rangle| \leq\|\mathrm{X}\|_{\mathbb{R}^{2}}\|\mathrm{Y}\|_{\mathbb{R}^{2}} .
$$


Se consideramos a função $f(x, y)$ definida por

$$
\left.\mathrm{f}(\mathrm{x}, \mathrm{y})=\left(\mathrm{x}^{2}+\mathrm{y}^{2}\right)\right)^{1 / 2}\left(\alpha^{2}+\beta^{2}\right)^{1 / 2}-\alpha \mathrm{x}-\beta \mathrm{y},
$$

poderemos usar o teste das segundas derivadas para mostrarmos que a função $\mathrm{f}(\mathrm{x}, \mathrm{y})$ é maior ou igual a zero, isto é, $f(x, y) \geq 0$. Daí segue a famosa desigualdade acima, que é essencial para estudar dualidade no $\mathbb{R}^{2}$. É interessante notar que $\mathrm{D}(\mathrm{a}, \mathrm{b})=0$, e o teste das segundas derivadas diz ser inconclusivo. $\dot{\mathrm{E}}$ importante ressaltar que fizemos o mesmo para as outras desigualdades e ilustraremos geometricamente todas as desigualdades. Um outro fato que ilustramos é que podemos considerar uma função de uma variável como se fosse uma função de duas variáveis, digamos

$$
f(x, y)=g(x) \quad \text { ou } \quad f(x, y)=h(y) .
$$

Veremos que $\mathrm{D}(\mathrm{a}, \mathrm{b})=0$, então o teste das segundas derivadas afirma ser inconclusivo onde as funções $\mathrm{g}(\mathrm{x})$ e $\mathrm{h}(\mathrm{y})$ são duas vezes diferenciáveis. No entanto, mostraremos via, um simples exemplo, que isso não é o caso. Também, ilustramos geometricamente.

Esses exemplos usando a teoria de máximo e mínimo de funções de duas variáveis para provar importantes desigualdades parece ser uma técnica nova e muito interessante. Assim, esses casos contradizem a afirmação do teste que diz $\mathrm{D}=0$ é inconclusivo. Em resumo, deixamos aqui essas notas para que os leitores possam tirar suas próprias conclusões.

Palavras-chave: Teste das segundas derivadas; desigualdades ;Young, Bernoulli; Cauchy-SchwarzBunyakowski.

\section{Abstract}

Finding local extrema in calculus requires knowledge of the so-called Second Derivative Test for one or two variables, often taught to students early in college, at least for those majoring in engineering, physics, or mathematics. In our experience teaching, we often tend to choose "nice academic" examples to illustrate the usefulness of the Second Derivative Test, and focusing less on the "not so nice" cases either because of time, or just because of our belief that students will not be interested in these often long and detailed examples. Our impetus in this short note is to bring the "not so nice" case or the pathological case of the Second Derivative Test for functions of two variables to the forefront and to illustrate its importance in connection to some inequalities often used in mathematical analysis.

Keywords: Second Derivative Test; Inequality; Young, Bernoulli; Cauchy-Schwarz-Bunyakowski

\section{Introdução}

Começaremos relembrando o conhecido teorema do teste da segunda derivada, frequentemente usado em livros de Cálculo, veja por exemplo [2]. Para funções de uma variável, o teste da segunda derivada, o leitor interessado poderá consultar o Teorema 4.7 em [2].

Teorema 1 (Teorema 12.14, p.855 em [2]). 
Seja $\mathrm{f}(\mathrm{x}, \mathrm{y})$ uma função de duas variáveis definida em um disco contendo o ponto (a,b), com derivadas parciais de segunda ordem continuas. Suponha $\mathrm{f}_{\mathrm{x}}(\mathrm{a}, \mathrm{b})=\mathrm{f}_{\mathrm{y}}(\mathrm{a}, \mathrm{b})=0$ e seja $\mathrm{D} \equiv$ $\mathrm{D}(\mathrm{a}, \mathrm{b})=\mathrm{f}_{\mathrm{xx}}(\mathrm{a}, \mathrm{b}) \cdot \mathrm{f}_{\mathrm{yy}}(\mathrm{a}, \mathrm{b})-\left[\mathrm{f}_{\mathrm{xy}}(\mathrm{a}, \mathrm{b})\right]^{2}$. Ent $\tilde{a} o$,

- Se $\mathrm{D}>0$ e $\mathrm{f}_{\mathrm{xx}}(\mathrm{a}, \mathrm{b})>0$, então $\mathrm{f}(\mathrm{a}, \mathrm{b})$ é o mínimo local para $\mathrm{f}$.

- $S e \mathrm{D}>0$ e $\mathrm{f}_{\mathrm{xx}}(\mathrm{a}, \mathrm{b})<0$, então $\mathrm{f}(\mathrm{a}, \mathrm{b})$ é o máximo local para $\mathrm{f}$.

- Se $\mathrm{D}<0$, então $\mathrm{f}(\mathrm{a}, \mathrm{b})$ é o ponto de sela para $\mathrm{f}$.

- Se $\mathrm{D}=0$, o teste é considerado inconclusivo.

Demonstração. O leitor interessado pode consultar o Apêndice B em [2].

O último caso é patológico no sentido de que muitas vezes não é muito claro que tipo de extremo obteremos em geral quando isso acontecer. Além disso, em muitos livros de cálculo, não há quase nenhum exemplo envolvendo essa situação. Acontece que muitas desigualdades interessantes estão conectadas a essa situação. A seguir, discutiremos respectivamente a desigualdade de Bernoulli, a desigualdade das médias aritméticas e geométricas, a desigualdade de CauchySchwarz-Bunyakowsky, a desigualdade de Young e sua relação com esse caso patológico. Também forneceremos representações pictóricas tridimensionais para ilustrar sua veracidade.

Começaremos com a desigualdade de Bernoulli, publicada por Jacob Bernoulli em 1669. Notamos, entretanto, que Joseph Hoffman (1969) a atribuiu à obra de Sluse (1668).

\section{Desigualdade de Bernoulli}

Teorema 2 (ver [1]). Seja $\mathrm{n}$ um inteiro positivo e $\mathrm{x}$ um número real tal que $\mathrm{x} \geq-1$. Então, a seguinte desigualdade mantém-se:

$$
(1+x)^{n} \geq 1+n x .
$$

Observação 1. Observamos que quando n é par, a desigualdade vale para todo número real x. Além disso, a desigualdade é estrita para $\mathrm{n} \geq 2$ e $\mathrm{x} \geq-1$ e $\mathrm{x} \neq 0$.

Observação 2 (O método do intervalo fechado. Veja a página 278 em [2]). Para encontrar os valores mínimos ou máximos absolutos de uma função contínua f em um intervalo fechado [a,b]:

1. Encontre os valores de f nos pontos críticos de $\mathrm{f}$ em $(\mathrm{a}, \mathrm{b})$.

2. Encontre os valores de f nas extremidades do intervalo [a,b].

3. O maior dos valores dos Passos 1 e 2 é o valor máximo absoluto de $\mathrm{f}$ em [a,b]. O menor desses valores é o valor mínimo absoluto de f em [a,b].

Essa desigualdade assume duas formas diferentes quando n é um número real positivo r. Na verdade, para $\mathrm{x} \geq-1$, temos respectivamente

$$
\begin{array}{ll}
(1+x)^{r} \geq 1+r x & , \quad \text { para } r \geq 1 \\
(1+x)^{r} \leq 1+r x & , \quad \text { para } 0 \leq r \leq 1
\end{array}
$$


Para provar essa desigualdade, vamos definir a função $f(x)=(1+x)^{n}-1-n x$. Essa função possui a derivada $\mathrm{f}^{\prime}(\mathrm{x})=\mathrm{n}(1+\mathrm{x}) \mathrm{n}-1-\mathrm{n}$ e os pontos críticos são soluções da equação $(1+\mathrm{x})^{\mathrm{n}-1}=1$. A única solução real é $\mathrm{x}=0$. Dado que $\mathrm{f}(0)=0$ e $\mathrm{f}^{\prime \prime}(0)=\mathrm{n}(\mathrm{n}-1)$, então o teste da segunda derivada para funções de uma variável implica que o ponto $(0,0)$ é o mínimo local por $n \geq 1$. Para provar que $(0,0)$ é um mínimo absoluto (ou global), ou seja, $\mathrm{f}(0) \leq \mathrm{f}(\mathrm{x})$ para todo $\mathrm{x} \geq-1$, procederemos por contradição. De fato, suponha que haja um $c \geq-1$ tal que $f(c)<f(0)$ para todos os $x \geq-1$. Então, usando a fórmula de extensão binomial de Newton, teremos

$$
(1+c)^{n}=\sum_{k=0}^{n}\left(\begin{array}{l}
n \\
k
\end{array}\right) c^{k}=1+n c+\frac{n(n-1)}{2} c^{2}+\cdots+c^{n}
$$

Consequentemente, se $\mathrm{c} \geq 0$ e $\mathrm{f}(\mathrm{c})<\mathrm{f}(0)$ implica $\frac{\mathrm{n}(\mathrm{n}-1)}{2} \mathrm{c}^{2}+\cdots+\mathrm{c}^{\mathrm{n}}<0$, o que é uma contradição óbvia, uma vez que todos os termos $c, c^{2}, \cdots, c^{n}$ são positivos.

Agora, suponha $c \in[-1,0]$. Observamos que a função $f(x)=(1+x)^{n}-1-n x$ é contínua no intervalo fechado $[-1,0]$. Além disso, $\mathrm{f}^{\prime}(0)=0$, i.e., 0 é um ponto crítico para a função $\mathrm{f}$. Observamos $f(-1)=n-1>0=f(0)$, para $n \geq 2$. Segue, usando o método do intervalo fechado acima, que o menor valor de $\mathrm{f}(\mathrm{x})$ no intervalo $[-1,0]$ é $\mathrm{f}(0)=0$, ou seja, $\mathrm{f}(0)$ é o mínimo absoluto de $\mathrm{f}(\mathrm{x})$ em $[-1,0]$. A Figura 1 abaixo é uma ilustração de $f(x)=(1+x)^{n}-1-n x$ para diferentes valores de $n$.

$$
f(x)=(1+x)^{n}-1-n x
$$

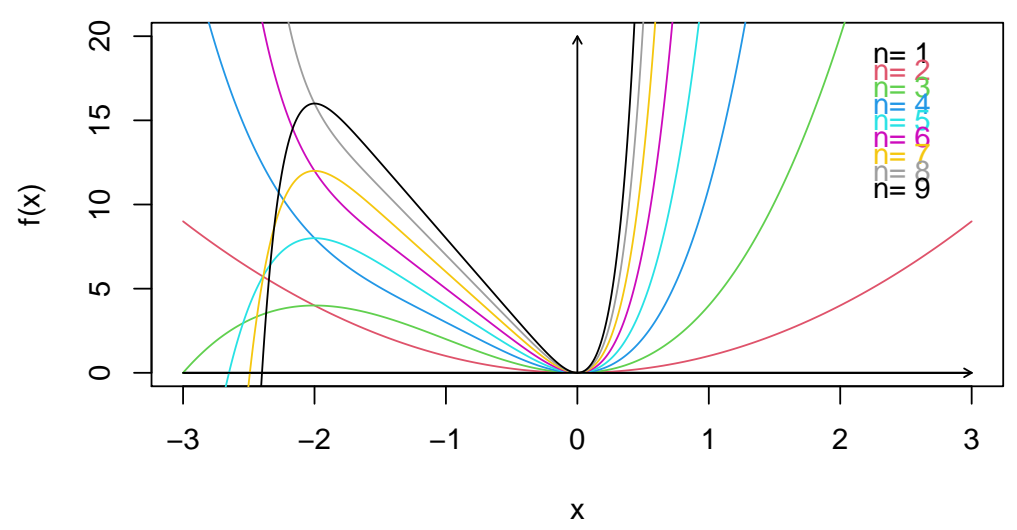

Figura 1: Ilustração de $\mathrm{f}(\mathrm{x})=(1+\mathrm{x})^{\mathrm{n}}-1-\mathrm{nx}$ para diferentes valores de $\mathrm{n}$.

Notamos, entretanto, que usando o teste da segunda derivada (Teorema 1) com $\mathrm{g}(\mathrm{x}, \mathrm{y})=(1+\mathrm{x})^{\mathrm{n}}-$ $1-\mathrm{nx}$, obteremos $\frac{\partial \mathrm{g}(\mathrm{x}, \mathrm{y})}{\partial \mathrm{x}}=\mathrm{n}(1+\mathrm{x})^{\mathrm{n}-1}-\mathrm{n}, \frac{\partial \mathrm{g}(\mathrm{x}, \mathrm{y})}{\partial \mathrm{y}}=0$, de modo que os pontos críticos sejam $(0, \mathrm{t})$ para qualquer $\mathrm{t} \geq 0$. Além disso, $\mathrm{D}(0, \mathrm{t})=0$ de forma que o caso patológico do teste da segunda derivada seja observado e nenhuma conclusão teria sido alcançada com ele. 
$n=3$

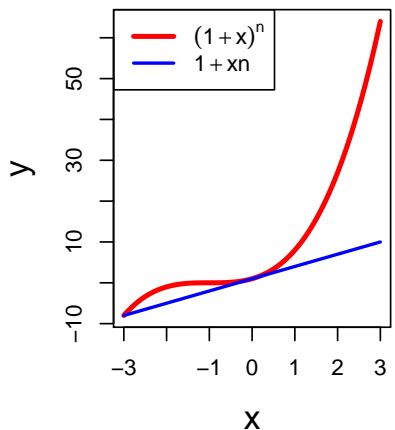

$\mathrm{n}=\mathbf{0 . 7 1}$

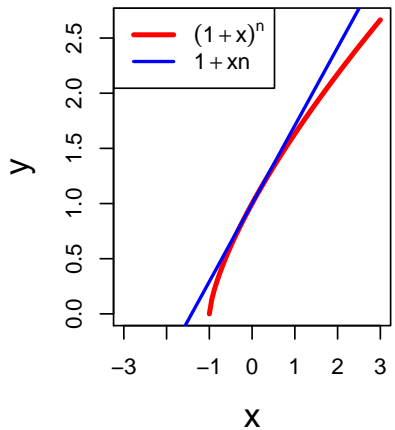

$n=4$

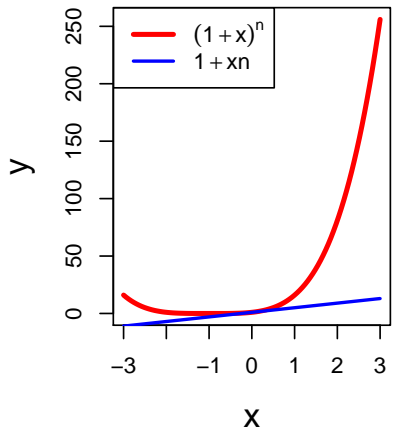

$\mathrm{n}=1.7$

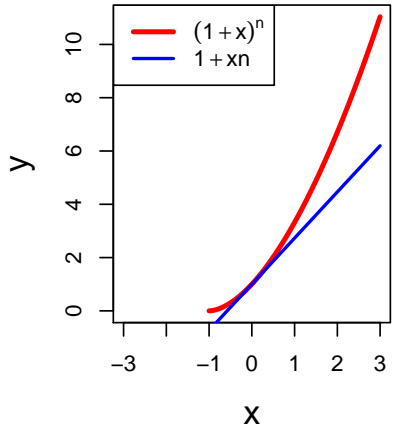

Figura 2: Esse gráfico mostra a relação entre $(1+\mathrm{x})^{\mathrm{n}}$ e $1+\mathrm{nx}$ para os valores $\mathrm{n}=3 ; 4 ; 0,71$ e 1,7 respectivamente

\section{Desigualdade entre as Médias Aritmética e Geométrica}

A seguir, discutiremos a relação entre as médias aritméticas e geométricas.

Teorema 3. Sejam $\mathrm{x}_{1}, \mathrm{x}_{2}, \cdots, \mathrm{x}_{\mathrm{n}}$ números reais positivos e $\mathrm{n}$ um inteiro positivo. Então, a seguinte desigualdade (AM-GM) é verdadeira:

$$
\frac{x_{1}+x_{2}+\cdots+x_{n}}{n} \geq \sqrt[n]{x_{1} \cdot x_{2} \cdots x_{n}}
$$

A igualdade é alcançada quando $\mathrm{x}_{1}=\mathrm{x}_{2}=\cdots=\mathrm{x}_{\mathrm{n}}$.

Observação 3. Vemos claramente que apesar de a desigualdade ser verdadeira apenas para números positivos $\mathrm{x}_{\mathrm{i}}$, a média aritmética existe mesmo quando os $\mathrm{x}_{\mathrm{i}}$ são de sinais mistos. A média geométrica encontra seu uso quando estamos lidando com quantidades absolutas. Há uma aplicação interessante dessa desigualdade em Ecologia, veja [5]. Para usar a terminologia ecológica, esse 
manuscrito mostra que as espécies invasoras têm valores de sensibilidade negativos ou positivos quando em modo de facilitação com outras espécies. Sua diferença média de nicho, portanto, só pode ser descrita pela média aritmética. No entanto, no modo de competição, seus valores de sensibilidade são todos positivos. Portanto, sua diferença média de nicho pode ser descrita pela média geométrica, com uma diferença de nicho aumentada (devido à desigualdade), que, em termos leigos, é benéfica para as espécies nativas.

Provaremos a seguir a desigualdade AM-GM para $n=2$. Considere a função $f(x, y)=\frac{x+y}{2}-\sqrt{x y}$, para $\mathrm{x}, \mathrm{y} \geq 0$. As primeiras derivadas parciais são dadas por

$$
\frac{\partial \mathrm{f}(\mathrm{x}, \mathrm{y})}{\partial \mathrm{x}}=\frac{1}{2}-\frac{1}{2} \sqrt{\frac{\mathrm{y}}{\mathrm{x}}}, \quad \frac{\partial \mathrm{f}(\mathrm{x}, \mathrm{y})}{\partial \mathrm{y}}=\frac{1}{2}-\frac{1}{2} \sqrt{\frac{\mathrm{x}}{\mathrm{y}}} .
$$

As segundas derivadas parciais são dadas por

$$
\frac{\partial^{2} \mathrm{f}(\mathrm{x}, \mathrm{y})}{\partial \mathrm{x}^{2}}=\frac{\sqrt{\mathrm{y}}}{4 \sqrt{\mathrm{x}^{3}}}, \quad \frac{\partial^{2} \mathrm{f}(\mathrm{x}, \mathrm{y})}{\partial \mathrm{x} \partial \mathrm{y}}=-\frac{1}{4 \sqrt{\mathrm{xy}}}, \quad \frac{\partial^{2} \mathrm{f}(\mathrm{x}, \mathrm{y})}{\partial \mathrm{y}^{2}}=\frac{\sqrt{\mathrm{x}}}{4 \sqrt{\mathrm{y}^{3}}} .
$$

Os pontos críticos são dados por

$$
\frac{\partial \mathrm{f}(\mathrm{x}, \mathrm{y})}{\partial \mathrm{x}}=0 \text { e } \frac{\partial \mathrm{f}(\mathrm{x}, \mathrm{y})}{\partial \mathrm{y}}=0 \Rightarrow \mathrm{x}=\mathrm{y} .
$$

Além disso, quando $\mathrm{x}=\mathrm{y}$, temos

$$
\frac{\partial^{2} \mathrm{f}(\mathrm{x}, \mathrm{x})}{\partial \mathrm{x}^{2}}=\frac{\partial^{2} \mathrm{f}(\mathrm{x}, \mathrm{x})}{\partial \mathrm{y}^{2}}=\frac{1}{4 \mathrm{x}}, \quad \frac{\partial^{2} \mathrm{f}(\mathrm{x}, \mathrm{x})}{\partial \mathrm{x} \partial \mathrm{y}}=-\frac{1}{4 \mathrm{x}} .
$$

Segue-se que $\mathrm{D}=\mathrm{D}(\mathrm{x}, \mathrm{x})=0$ e, portanto, estamos no caso patológico do teste da segunda derivada. Mostraremos que $(\mathrm{x}, \mathrm{x})$ é local, na verdade um mínimo local. Mostraremos que uma simples prova por contradição será suficiente. De fato, suponha que haja um ponto $(a, b)$ tal que $f(a, b)<f(x, x)$. Ou seja,

$$
\frac{a+b}{2}-\sqrt{a b}<\frac{x+x}{2}-\sqrt{x^{2}}=0
$$

Isso significa $\frac{a+b}{2}-\sqrt{a b}<0$. Então, elevando os dois lados ao quadrado, temos que $(a+b)^{2}<$ $(2 \sqrt{a b})^{2}$, ou seja, $a^{2}+2 a b+b^{2}<4 a b \Rightarrow a^{2}-2 a b+b^{2}<0 \Rightarrow(a b)^{2}<0$, o que é uma contradição.

Também podemos observar que a contradição pode ser obtida observando que

$$
\frac{a+b}{2}-\sqrt{a b}<0 \Longleftrightarrow a+b-2 \sqrt{a b}<0 \Longleftrightarrow(\sqrt{a}-\sqrt{b})^{2}<0 \text {. }
$$

Portanto, $f(a, b) \geq 0$ para todos $a, b \geq 0$, ou seja,

$$
\sqrt{a b} \leq \frac{a+b}{2} .
$$




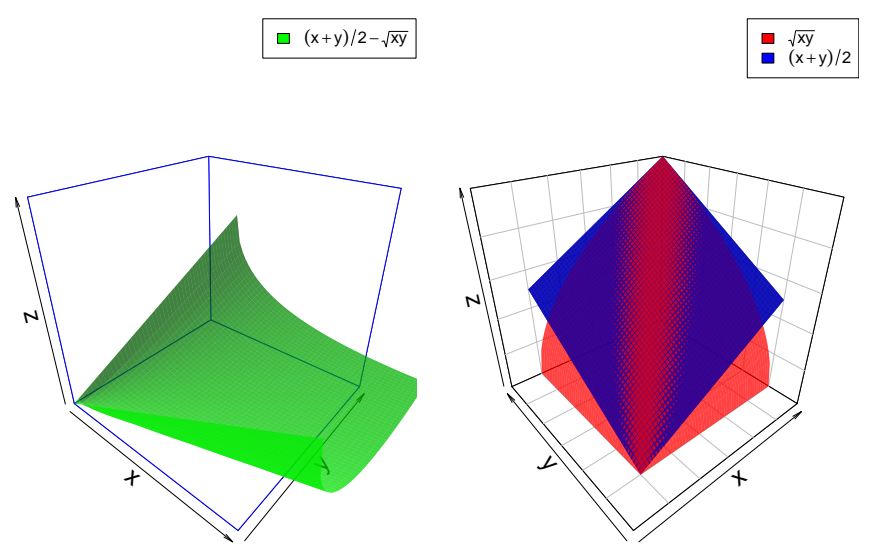

Figura 3: O primeiro gráfico mostra a superfície $(\mathrm{x}+\mathrm{y}) / 2-\sqrt{\mathrm{xy}}$ (verde) ficando acima do nível $\mathrm{z}=0$, e o segundo gráfico mostra a superfície $(\mathrm{x}+\mathrm{y}) / 2$ (azul) sempre acima da superfície $\sqrt{\mathrm{xy}}$ (vermelho).

A seguir, discutimos uma desigualdade devida a William Henry Young (1912).

\section{Desigualdade de Young}

Sejam p e q números positivos que satisfazem $\frac{1}{\mathrm{p}}+\frac{1}{\mathrm{q}}=1$. Se a e b são números reais não negativos, o seguinte é verdadeiro:

$$
a b \leq \frac{a^{p}}{p}+\frac{b^{q}}{q} .
$$

A igualdade ocorre quando $\mathrm{a}^{\mathrm{p}}=\mathrm{b}^{\mathrm{q}}$.

Existem numerosas generalizações dessa desigualdade, devido a Young, ver [8]. Abaixo, mencionamos uma que é particularmente útil na análise funcional.

Teorema 4. Seja f uma função contínua crescente definida para números reais não negativos $\mathrm{x}$, com $\mathrm{f}(0)=0$. Suponha que a, b sejam números reais positivos, de modo que a esteja no domínio de $\mathrm{f}$ e b esteja na sua imagem. Então, o seguinte é verdadeiro:

$$
a b \leq \int_{0}^{a} f(x) d x+\int_{0}^{b} f^{-1}(x) d x
$$

onde $\mathrm{f}^{-1}$ é a função inversa de $\mathrm{f}$.

Essa desigualdade pode ser interpretada como: a soma das áreas de f no intervalo $(0, a)$ e de $\mathrm{f}^{-1}$ no intervalo $(0, b)$ é pelo menos a área do retângulo de base a e altura b; veja a Fig. 4 abaixo. 
Notamos que se tomarmos $\mathrm{f}(\mathrm{x})=\mathrm{x}^{\mathrm{p}-1}$ em (6), então $\mathrm{f}(\mathrm{x})=\mathrm{x}^{\mathrm{q}-1}$ e iremos recuperar a desigualdade em (5).

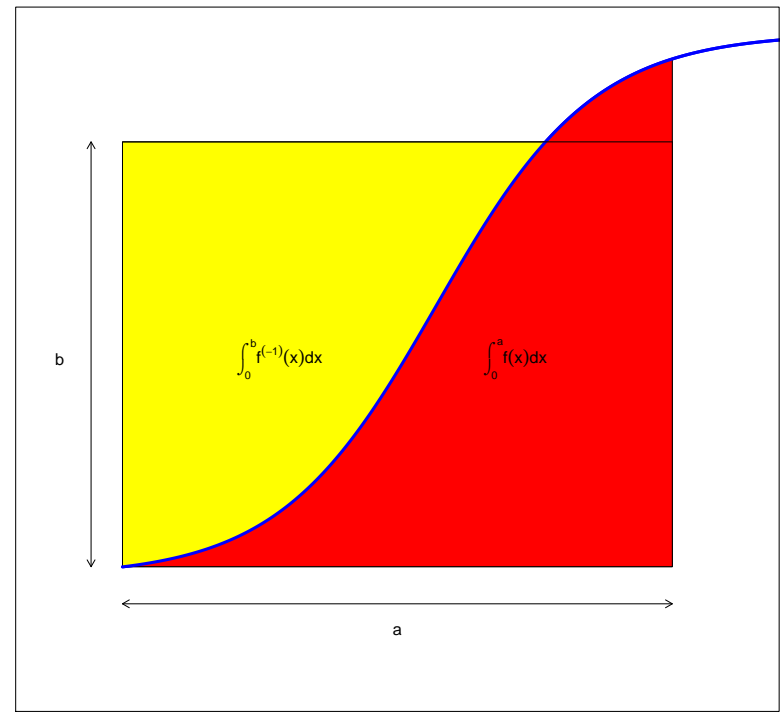

Figura 4: A soma das duas áreas (em amarelo e vermelho) é pelo menos a área do retângulo com base a e altura b

Conectaremos a desigualdade de Young ao teste da segunda derivada para $\mathrm{p}=\mathrm{q}=2$. Defina a função $f(x, y)=\frac{x^{2}}{2}+\frac{y^{2}}{2}-x y$, para $x, y>0$. Notamos que $f$ é contínua e duas vezes diferenciável em $\mathrm{x}$ e $\mathrm{y}$ e as derivadas parciais de primeira ordem são:

$$
\frac{\partial \mathrm{f}(\mathrm{x}, \mathrm{y})}{\partial \mathrm{x}}=\mathrm{x}-\mathrm{y}, \quad \frac{\partial \mathrm{f}(\mathrm{x}, \mathrm{y})}{\partial \mathrm{y}}=\mathrm{y}-\mathrm{x} .
$$

Portanto, os pontos críticos encontram-se na linha $\mathrm{x}=\mathrm{y}$. As segundas derivadas parciais são dadas por

$$
\frac{\partial^{2} \mathrm{f}(\mathrm{x}, \mathrm{y})}{\partial \mathrm{x}^{2}}=\frac{\partial^{2} \mathrm{f}(\mathrm{x}, \mathrm{y})}{\partial \mathrm{y}^{2}}=1, \quad \frac{\partial^{2} \mathrm{f}(\mathrm{x}, \mathrm{y})}{\partial \mathrm{x} \partial \mathrm{y}}=-1 .
$$

Segue-se que $\mathrm{D}(\mathrm{x}, \mathrm{x})=1^{2}-1=0$ e, portanto, novamente, temos o caso patológico do teste da segunda derivada.

No entanto, mostraremos usando a contradição que $0 \leq \mathrm{f}(\mathrm{x}, \mathrm{y})$, para todos $\mathrm{x}, \mathrm{y}>0$. Suponha que não seja esse o caso, ou seja, há um ponto $\mathrm{x}_{0}, \mathrm{y}_{0} \operatorname{com~} \mathrm{x}_{0}, \mathrm{y}_{0}>0$ tal que $\mathrm{f}\left(\mathrm{x}_{0}, \mathrm{y}_{0}\right)<0$. Isso implicará que

$$
\left(\frac{\mathrm{x}_{0}^{2}}{2}+\frac{\mathrm{y}_{0}^{2}}{2}\right)^{2}<\left(\mathrm{x}_{0} \mathrm{y}_{0}\right)^{2} \text {. }
$$


Consequentemente, teremos que $\left(\mathrm{x}_{0}^{2}-\mathrm{y}_{0}^{2}\right)^{2}<0$, que é uma contradição. Assim, temos $\mathrm{f}(\mathrm{x}, \mathrm{y}) \geq 0$ para todos $\mathrm{x}, \mathrm{y}>0$, ou seja,

$$
\mathrm{xy} \leq \frac{\mathrm{x}^{2}}{2}+\frac{\mathrm{y}^{2}}{2}, \quad \text { para todos } \mathrm{x}, \mathrm{y}>0
$$

A Figura 5 abaixo é uma ilustração desse resultado em três dimensões.

$$
\square x^{2} / 2+y^{2} / 2-x y
$$
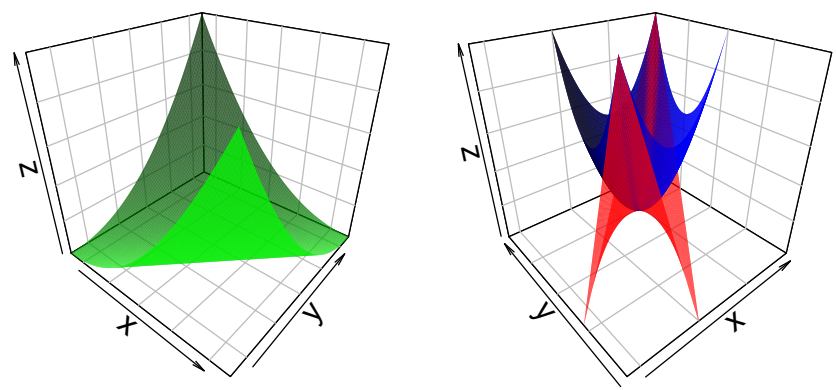

Figura 5: O primeiro gráfico mostra a superfície $\mathrm{x}^{2} / 2+\mathrm{y}^{2} / 2-\mathrm{xy}$ (verde) ficando acima do nível $\mathrm{z}=0$, e o segundo gráfico mostra a superfície $\mathrm{x}^{2} / 2+\mathrm{y}^{2} / 2$ (azul) sempre acima da superfície xy (vermelho).

Agora, apresentamos a célebre e amplamente utilizada desigualdade de Cauchy-Schwarz-Bunyakowski, derivada pela primeira vez por Augustin Louis Cauchy (1821).

\section{Desigualdade de Cauchy-Schwarz-Bunyakowsky}

Sejam $\mathrm{x}_{1}, \mathrm{x}_{2}, \cdots \mathrm{x}_{\mathrm{n}}>0$ e $\mathrm{y}_{1}, \mathrm{y}_{2}, \cdots, \mathrm{y}_{\mathrm{n}}>0$. A seguinte desigualdade é verdadeira:

$$
\sum_{i=1}^{n} x_{i} y_{i} \leq \sqrt{\sum_{i=1}^{n} x_{i}^{2}} \cdot \sqrt{\sum_{i=1}^{n} y_{i}^{2}} .
$$

A igualdade é alcançada quando $\mathrm{x}_{\mathrm{i}}$ e $\mathrm{y}_{\mathrm{i}}$ são proporcionais e foi estabelecida por Cauchy [4].

Existem várias generalizações desses resultados. Começamos com aquele dado por Herman Schwarz em [7] para espaços de produtos internos:

Teorema 5. Seja $\mathrm{V}$ um espaço de produto interno e sejam $\mathrm{x}=\left(\mathrm{x}_{1}, \mathrm{x}_{2}, \cdots, \mathrm{x}_{\mathrm{n}}\right), \mathrm{y}=\left(\mathrm{y}_{1}, \mathrm{y}_{2}, \cdot, \mathrm{y}_{\mathrm{n}}\right)$ em $\mathrm{V}$. Defina o produto escalar de $\mathrm{x}$ e $\mathrm{y}$ como $\langle\mathrm{x}, \mathrm{y}\rangle$. Defina a norma de $\mathrm{x}$ em $\mathrm{V}$ como $\|\mathrm{x}\|_{\mathrm{V}}=\langle\mathrm{x}, \mathrm{x}\rangle^{\frac{1}{2}}$. 
A seguinte desigualdade é verdadeira:

$$
|\langle\mathrm{x}, \mathrm{y}\rangle| \leq\|\mathrm{x}\|_{\mathrm{V}} \cdot\|\mathrm{y}\|_{\mathrm{V}}
$$

Na verdade, se $V=\mathbb{R}^{n}$, então $\langle x, y\rangle=\sum_{i=1}^{n} x_{i} y_{i}, e, c o m\|x\|_{V}=\left(\sum_{i=1}^{n}\left|x_{i}\right|^{2}\right)^{1 / 2}$, obteremos a desigualdade em (7). Também mencionamos uma generalização devida a Victor Yakovlevich Bunyakowsky [3], que derivou uma desigualdade semelhante para integrais.

Teorema 6. Sejam $\mathrm{f}$ e $\mathrm{g}$ duas funções quadrado integráveis em $\mathbb{R}^{\mathrm{n}}$. Então, o seguinte é verdadeiro:

$$
\left|\int_{\mathbb{R}^{n}} f(x) g(x) d x\right| \leq\left(\int_{\mathbb{R}^{n}} f^{2}(x) d x\right)^{1 / 2} \cdot\left(\int_{\mathbb{R}^{n}} g^{2}(x) d x\right)^{1 / 2} .
$$

Observamos que, no teorema acima, $V=\mathrm{L}^{2}\left(\mathbb{R}^{\mathrm{n}}\right.$ ) (o espaço de funções quadrado integráveis em $\mathbb{R}^{\mathrm{n}}$ ) é um espaço de produto interno com um produto interno definido como

$$
\langle f, g\rangle=\int_{\mathbb{R}^{n}} f(x) g(x) d x \quad \text { e } \quad\|f\|_{V}=\left(\int_{\mathbb{R}^{n}} f^{2}(x) d x\right)^{1 / 2} .
$$

Outra generalização, que foi mencionada pela primeira vez por Leonard James Rogers (1888) e posteriormente derivada por Otto Ludwig Hölder em 1889, é fornecida abaixo:

Teorema 7. Sejam p e q dois números reais tais que $\frac{1}{\mathrm{p}}+\frac{1}{\mathrm{q}}=1$. Então, o seguinte é verdadeiro:

$$
\sum_{i=1}^{n} x_{i} y_{i} \leq\left(\sum_{i=1}^{n} x_{i}^{p}\right)^{1 / p} \cdot\left(\sum_{i=1}^{n} x_{i}^{q}\right)^{1 / q}
$$

Observação 4. A desigualdade em (7) é obviamente obtida tomando $\mathrm{p}=\mathrm{q}=2 \mathrm{em}$ (9). Seria uma tarefa tola tentar enumerar todas as aplicações dessa desigualdade. No entanto, vamos mencionar uma útil na área de Estatística:

Sejam $a=\left(a_{1}, \cdots, a_{n}\right)$ e $b=\left(b_{1}, \cdots, b_{n}\right)$ dois vetores de números reais, com as respectivas médias aritméticas $\overline{\mathrm{a}}$ e $\overline{\mathrm{b}}$. A covariância entre $\mathrm{a}$ e $\mathrm{b}$ é definida como

$$
\operatorname{Cov}(a, b)=\frac{1}{n-1} \sum_{i=1}^{n}\left(a_{i}-\bar{a}\right)\left(b_{i}-\bar{b}\right) .
$$

Essa quantidade foi introduzida pela primeira vez por Karl Pearson em [6] como uma medida de associação linear entre os vetores a e b. Essencialmente, uma covariância grande/pequena significa uma associação linear forte/fraca. Além disso, uma covariância positiva/negativa também significa uma associação positiva/negativa entre a e b. Acontece, entretanto, que a covariância Cov(a,b) é uma quantidade que pode assumir valores arbitrários e, portanto, não é muito sábio usá-la para quantificar o grau de associação linear entre dois vetores a e b. Além disso, uma mudança de unidade entre a e b afetaria drasticamente $\operatorname{Cov}(\mathrm{a}, \mathrm{b})$. Para corrigir essas falhas, Karl Pearson propôs dividir $\operatorname{Cov}(a, b)$ por uma constante de normalização $\sqrt{\operatorname{Cov}(a, a)} \cdot \sqrt{\operatorname{Cov}(b, b)}$ e, portanto, a quantidade resultante é o que é conhecido hoje como o coeficiente de correlação de Pearson, definido como

$$
R(a, b)=\frac{\sum_{i=1}^{n}\left(a_{i}-\bar{a}\right)\left(b_{i}-\bar{b}\right)}{\sqrt{\sum_{i=1}^{n}\left(a_{i}-\bar{a}\right)^{2}} \sqrt{\sum_{i=1}^{n}\left(b_{i}-\bar{b}\right)^{2}}} .
$$


Usando a desigualdade de Cauchy-Schwarz-Bunyakowsky como em (7) com $x_{i}=a_{i}-\bar{a}$ e $y_{i}=b_{i}-\bar{b}$, segue-se que $|R(a, b)| \leq 1$. A igualdade é alcançada quando a e b são proporcionais ou, em termos leigos, quando a e b são perfeitamente correlacionados. Além disso, R(a,b) é uma quantidade adimensional e, portanto, não contém unidades.

Existem várias provas dessa desigualdade, incluindo a prova original de Cauchy em que ele reescreve (7) de uma forma que é obviamente verdadeira:

$$
0 \leq \sum_{1 \leq \mathrm{i} \leq \mathrm{j} \leq \mathrm{n}}\left(\mathrm{x}_{\mathrm{i}} \mathrm{x}_{\mathrm{j}}-\mathrm{x}_{\mathrm{j}} \mathrm{x}_{\mathrm{i}}\right)^{2}
$$

Daremos uma prova dessa desigualdade, para $n=2$ em conexão com o Teste da Segunda Derivada. Seja $f(x, y)=\sqrt{a^{2}+b^{2}} \sqrt{x^{2}+y^{2}}-a x-b x$. Notamos que $f$ é duas vezes diferenciável e as derivadas parciais de primeira ordem são fornecidas como

$$
\frac{\partial \mathrm{f}(\mathrm{x}, \mathrm{y})}{\partial \mathrm{x}}=\frac{\mathrm{x} \sqrt{\mathrm{a}^{2}+\mathrm{b}^{2}}}{\sqrt{\mathrm{x}^{2}+\mathrm{y}^{2}}}-\mathrm{a}, \quad \frac{\partial \mathrm{f}(\mathrm{x}, \mathrm{y})}{\partial \mathrm{y}}=\frac{\mathrm{y} \sqrt{\mathrm{a}^{2}+\mathrm{b}^{2}}}{\sqrt{\mathrm{x}^{2}+\mathrm{y}^{2}}}-\mathrm{b} .
$$

Segue-se que os pontos críticos são soluções da equação $\mathrm{x}=\frac{\mathrm{a}}{\mathrm{b}} \mathrm{y}$, ou seja, os pontos $\left(\frac{\mathrm{a}}{\mathrm{b}} \mathrm{y}, \mathrm{y}\right)$. Da mesma forma, as derivadas parciais de segunda ordem são:

$$
\begin{aligned}
& \frac{\partial^{2} \mathrm{f}(\mathrm{x}, \mathrm{y})}{\partial \mathrm{x}^{2}}=\sqrt{\mathrm{a}^{2}+\mathrm{b}^{2}}\left(\frac{1}{\sqrt{\mathrm{x}^{2}+\mathrm{y}^{2}}}-\frac{\mathrm{x}^{2}}{\sqrt{\left(\mathrm{x}^{2}+\mathrm{y}^{2}\right)^{3}}}\right), \\
& \frac{\partial^{2} \mathrm{f}(\mathrm{x}, \mathrm{y})}{\partial \mathrm{y}^{2}}=\sqrt{\mathrm{a}^{2}+\mathrm{b}^{2}}\left(\frac{1}{\sqrt{\mathrm{x}^{2}+\mathrm{y}^{2}}}-\frac{\mathrm{y}^{2}}{\sqrt{\left(\mathrm{x}^{2}+\mathrm{y}^{2}\right)^{3}}}\right), \\
& \frac{\partial^{2} \mathrm{f}(\mathrm{x}, \mathrm{y})}{\partial \mathrm{x} \partial \mathrm{y}}=-\frac{\mathrm{xy} \sqrt{\mathrm{a}^{2}+\mathrm{b}^{2}}}{\sqrt{\left(\mathrm{x}^{2}+\mathrm{y}^{2}\right)^{3}}} .
\end{aligned}
$$

Observe que $\mathrm{D}\left(\frac{\mathrm{a}}{\mathrm{b}} \mathrm{y}, \mathrm{y}\right)=0$, e mais uma vez, obtemos o caso patológico do teste da segunda derivada. Mostraremos por contradição que $\mathrm{f}(\mathrm{x}, \mathrm{y}) \geq 0$ para todo $\mathrm{x}, \mathrm{y} \in \mathbb{R}$. Sem perda de generalidade, provaremos o caso com x, y $>0$, e o procedimento é semelhante para os outros casos. Se houver $\mathrm{x}_{0}, \mathrm{y}_{0}>0$ tais que $\mathrm{f}\left(\mathrm{x}_{0}, \mathrm{y}_{0}\right)<0$, então teríamos $\sqrt{\mathrm{a}^{2}+\mathrm{b}^{2}} \sqrt{\mathrm{x}_{0}^{2}+\mathrm{y}_{0}^{2}}<\mathrm{ax}_{0}+$ por $_{0}$. Quadrando ambos os lados, temos $\left(\mathrm{a}^{2}+\mathrm{b}^{2}\right)\left(\mathrm{x}_{0}^{2}+\mathrm{y}_{0}^{2}\right)<\left(\mathrm{ax}_{0}+\mathrm{by}_{0}\right)^{2}$. Expandindo ambos os lados desse lado da desigualdade, temos: $\mathrm{a}^{2} \mathrm{x}_{0}^{2}+\mathrm{b}^{2} \mathrm{x}_{0}^{2}+\mathrm{a}^{2} \mathrm{y}_{0}^{2}+\mathrm{b}^{2} \mathrm{y}_{0}^{2}<\mathrm{a}^{2} \mathrm{x}_{0}^{2}+2 a b \mathrm{x}_{0} \mathrm{y}_{0}+\mathrm{b}^{2} \mathrm{y}_{0}^{2}$. Após a simplificação, obtemos: $\mathrm{b}^{2} \mathrm{x}_{0}^{2}+\mathrm{a}^{2} \mathrm{y}_{0}^{2}-2 \mathrm{abx}_{0} \mathrm{y}_{0}<0$, que é equivalente $\mathrm{a}\left(\mathrm{bx}_{0}-\mathrm{ay}_{0}\right)^{2}<0$. Essa é uma contradição óbvia e, portanto, para todos os $\mathrm{x}, \mathrm{y}>0$, temos $\mathrm{f}(\mathrm{x}, \mathrm{y}) \geq 0$, ou seja, $\mathrm{ax}+$ por $\leq \sqrt{\mathrm{a}^{2}+\mathrm{b}^{2}} \sqrt{\mathrm{x}^{2}+\mathrm{y}^{2}}$.

De fato, mostramos que para $u=(a, b), v=(x, y)$, temos $|\langle u, v\rangle| \leq\|u\|\|v\|$ para $\|v\|=\left(x^{2}+y^{2}\right)^{1 / 2}$. A Figura 6 abaixo é uma ilustração desse fato para $\mathrm{a}=\mathrm{b}=1$. Notamos que a contradição é obtida usando um argumento semelhante à prova original de Cauchy usada. 

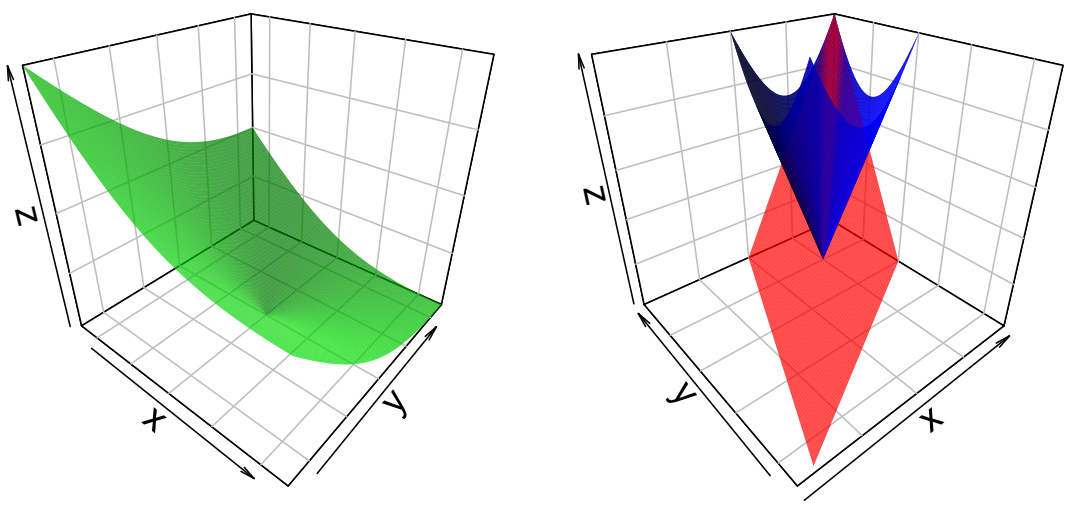

Figura 6: O primeiro gráfico mostra a superfície $\sqrt{2\left(\mathrm{x}^{2}+\mathrm{y}^{2}\right)}-\mathrm{x}-\mathrm{y}$ (verde) ficando acima do nível $\mathrm{z}=0$, e o segundo gráfico mostra a superfície $\sqrt{2\left(\mathrm{x}^{2}+\mathrm{y}^{2}\right)}$ (azul) sempre acima da superfície $\mathrm{x}+\mathrm{y}$ (vermelho).

\section{Discussão}

Nesta seção, gostaríamos de discutir os tipos de funções para as quais $\mathrm{D}(\mathrm{a}, \mathrm{b})=0$. Afirmamos que o conjunto de funções para as quais $\mathrm{D}(\mathrm{a}, \mathrm{b})=0$ é muito grande. De fato, considere qualquer função de uma variável única $\mathrm{g}(\mathrm{x})$ que seja duas vezes diferenciável. Defina $\mathrm{f}(\mathrm{x}, \mathrm{y})=\mathrm{g}(\mathrm{x})$, para todos os x no domínio de g e para todos os $\mathrm{y} \in \mathbb{R}$. Então, temos o seguinte:

$$
\frac{\partial^{2} \mathrm{f}(\mathrm{x}, \mathrm{y})}{\partial \mathrm{x}^{2}}=\mathrm{g}^{\prime \prime}(\mathrm{x}) ; \quad \frac{\partial^{2} \mathrm{f}(\mathrm{x}, \mathrm{y})}{\partial \mathrm{y}^{2}}=0 ; \quad \frac{\partial^{2} \mathrm{f}(\mathrm{x}, \mathrm{y})}{\partial \mathrm{x} \partial \mathrm{y}}=0 .
$$

Portanto,

$$
\mathrm{D}=\frac{\partial^{2} \mathrm{f}(\mathrm{x}, \mathrm{y})}{\partial \mathrm{x}^{2}} \cdot \frac{\partial^{2} \mathrm{f}(\mathrm{x}, \mathrm{y})}{\partial \mathrm{y}^{2}}-\left[\frac{\partial^{2} \mathrm{f}(\mathrm{x}, \mathrm{y})}{\partial \mathrm{x} \partial \mathrm{y}}\right]^{2}=0 .
$$

Isso prova que o caso $\mathrm{D}=0$ ocorre em muitas situações na prática.

Exemplo: Seja $f(x, y)=g(x)=x^{2}-4 x$. Então $g^{\prime}(x)=2 x-4 \Longrightarrow g^{\prime}(x)=0 \Longleftrightarrow x=2$.

Além disso, $\mathrm{g}^{\prime \prime}(\mathrm{x})=2>0$. Os pontos críticos para $\mathrm{f}(\mathrm{x}, \mathrm{y})$ são $(2, \mathrm{y})$ para todos os $\mathrm{y} \in \mathbb{R}$. Além disso, $\mathrm{f}(2, \mathrm{y})=-4$ e $\mathrm{D}(2, \mathrm{y})=0$, que é o caso patológico do Segundo Teste Derivado. Mostraremos 
que, de fato, $(2, y)$ é um mínimo global de $\mathrm{f}(\mathrm{x}, \mathrm{y})$. Suponha por contradição que existe $(\mathrm{a}, \mathrm{b}) \in \mathbb{R}^{2}$ tal que $\mathrm{f}(\mathrm{a}, \mathrm{b})<\mathrm{f}(2, \mathrm{y})$ para algum $\mathrm{y} \in \mathbb{R}$. Então teríamos $\mathrm{a}^{2}-4 \mathrm{a}<-4$, ou seja, $(\mathrm{a}-2)^{2}<0$ o que é impossível. Segue-se que $\mathrm{f}(\mathrm{a}, \mathrm{b}) \geq \mathrm{f}(2, \mathrm{y})=-4$ para todos $\mathrm{y} \in \mathbb{R}$, e para todos $(\mathrm{a}, \mathrm{b}) \in \mathbb{R}^{2}$ ou seja, -4 é um mínimo absoluto de f(x,y), conforme ilustrado na Figura 7 abaixo.
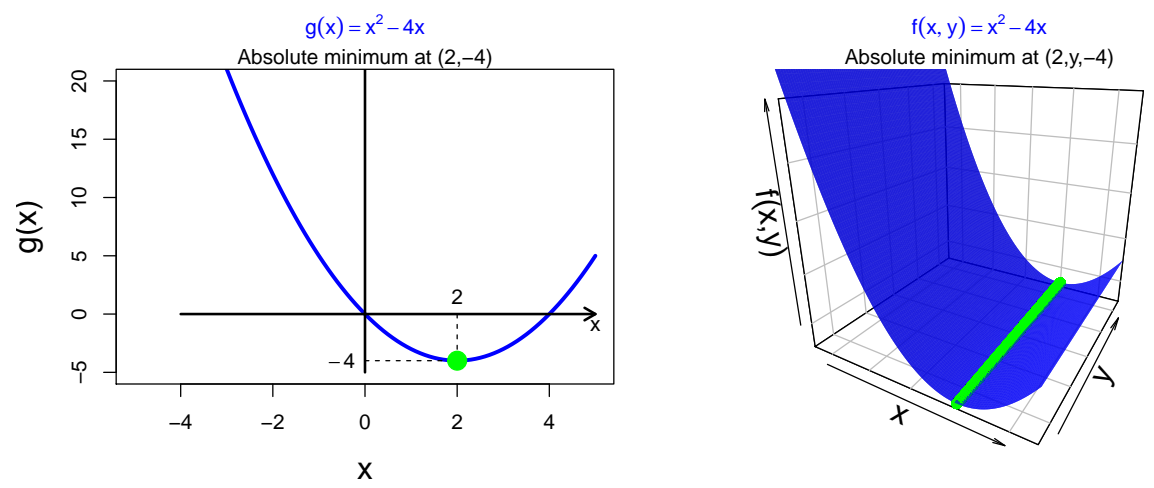

Figura 7: A figura à esquerda mostra que -4 é um mínimo absoluto para $\mathrm{g}(\mathrm{x})$ e o da direita mostra que $\mathrm{f}(2, \mathrm{y})=-4$ para todos $\mathrm{y} \in \mathbb{R}$ é um mínimo absoluto para $\mathrm{f}(\mathrm{x}, \mathrm{y})$.

Podemos concluir observando que, em geral, $\mathrm{g}(\mathrm{a})=\mathrm{b})$ é um extremo local/absoluto de g(x); então, $\mathrm{f}(\mathrm{a}, \mathrm{y})=\mathrm{b}$ é um extremo local $/$ absoluto de $\mathrm{f}(\mathrm{x}, \mathrm{y})=\mathrm{g}(\mathrm{x})$ onde $\mathrm{D}(\mathrm{a}, \mathrm{y})=0$, para todos $\mathrm{y} \in \mathbb{R}$.

\section{Conclusão}

Nesta breve nota, fornecemos exemplos de casos patológicos do teste da segunda derivada e suas relações com desigualdades conhecidas. Isso não é de forma alguma uma cobertura exaustiva de todas as desigualdades úteis. Podemos, no entanto, mencionar que as mesmas técnicas podem ser aplicadas à desigualdade de Minkowski, mas por uma questão de brevidade, ela foi omitida aqui. Esse esforço deve ser continuado para trazer noções complicadas em matemática a níveis que sejam mais atraentes para o público em geral.

\section{Agradecimentos}

Os autores gostariam de agradecer aos pareceristas anônimos por seus comentários e sugestões que ajudaram muito a melhorar a qualidade e o layout do artigo. Este trabalho foi originalmente escrito em inglês, e a tradução foi feita pelo professor Ronaldo Garcia.

\section{Referências}

[1] J. Bernoulli. Positiones arithmeticae de seriebus infinitis. Basel, 1689.

[2] W. Briggs and L. Cochran. Calculus: Early transcendentals. Addison-Wesley, 2010.

[3] V. Y. Bunyakowski. Sur quelques inégalités concernant les intégrales aux différences finies. Mémoires de l'Adadémie des sciences de St-Pétersbourg, 4:1-9, 1859.

[4] L. A. Cauchy. Cours sur l'analyse de l'École Royale Polytechnique, Première Partie, Analyse Algébrique. Debure frères, 1821. 
[5] E. E. Tansey, K. Schultz, C. Afferbach, K. Kwessi, K. Lyons, and E. Elaydi. From competition to facilitation: Expanding on mechanistic models of species interactions. Letters in Ecology, 2017.

[6] K. Pearson. Notes on regression and inheritance in the case of two parents. Proceedings of the Royal Society of London, 58:240-242, 1895.

[7] H. A. Schwarz. Über ein flächen kleinsten flächeninhalts betreffendes problem der variationsrechnung. Acta. Soc. Sci. Fennisca, 15:315-362, 1885.

[8] W. H. Young. On classes of summable functions and their Fourier series. Proceeding of the Royal Society of London, Series A, 87(594):225-229, 1912.

Eddy Kwessi

Department of Mathematics

Trinity University, San Antonio, TX, USA

<ekwessi@trinity.edu>

Seth Kermausuor

Department of Mathematics and Computer Science Alabama State University, USA <skermausour@alasu.edu>

Geraldo de Souza Department of Mathematics and Statistics Auburn University Auburn Alabama, USA <desougs@auburn.edu>

Recebido: $13 / 03 / 2020$ Publicado: 22/10/2020 